\title{
Abstracts of the \\ 4th Canadian Neuro-Oncology Meeting
}

\section{Summary}

In June, 1990, at the Hotel Fort Garry in Winnipeg, 57 clinicians and basic scientists from across North America met for the fourth time to update their progress and frustrations in the continuing study of malignant brain tumors. Thirty platform presentations and 7 posters, equally divided between basic and clinical material, were presented. Four invited guest speakers critically presented their area of expertise and spearheaded the ongoing discussions of each of the platform presentations.

Dr. Patrick Kelly (Minneapolis) reviewed the evolution of his work on computerassisted volumetric resection of brain tumors, which included a very thorough presentation and group discussion on stereotactic radiosurgery. Dr. Mark Rosenblum (San Francisco) highlighted his work and reviewed the field of tumor cell biology. Dr. John Kepes (Kansas City) reviewed his exhaustive experience with meningiomas, emphasizing patterns of regrowth and reviewing the many histologic variants. Dr. Douglas Arnold (Montreal) clarified the current spectrum of the usage of magnetic resonance imaging in the clinical evaluation of malignant brain tumors.

The majority of presentations were from individuals and laboratories who have presented at past meetings and who were commenting on the ongoing evolution of their clinical and basic science studies. A great deal of camaraderie and scientific cooperation has occurred as a result of these four meetings. The 5th Canadian Neuro-Oncology meeting will be held in Toronto in 1992.

The Organizing Committee 


\section{The 4th Canadian Neuro-oncology Meeting, 1990}

1.

Treatment of Malignant Glioma by Stereotaxic Implantation of Removable, High-Activity, lodine-125 Sources: An Update

M. MALKIN, E. ARBIT, L. ANDERSON, M. ROSENBLUM, D. FASS, J. GALICICH and S. LEIBEL (New York, U.S.A.)

Removable catheters, containing high-activity I-125 sources, were placed into malignant gliomas in 47 patients, using software to optimize radiation dosimetry and a Brown-Roberts-Wells stereotaxic system. There were 27 men and 20 women, age 7-73. Nine had recurrent anaplastic astrocytoma, and 38 glioblastoma. Median Karnofsky score was 80 , range 50-100. Twenty-nine patients were implanted at recurrence following resection, extemal irradiation and chemotherapy, and 18 after resection before further therapy. A dose of $60 \mathrm{~Gy}$ was delivered to the enhancing tumour contour on $\mathrm{CT}$ scan. Median dose rate was 35.7 cGy/hr. Tumour volumes were 6-187 cc, and implanted activity 79-495 $\mathrm{mCi}$. Of 29 patients treated at recurrence, 16 are alive; median survival from brachytherapy is 9.9 months. Of 18 patients treated after resection alone, 8 are alive; median survival 22.1 months. Four of these 8 are alive more than 12 months after implantation. The most common difficulty with the procedure was catheter misplacement, which occurred in 5 patients. Sixteen patients required 17 reoperations, 1-25 months after implantation, for an enhancing mass at the operative site. Tumour alone was found in 2, radiation necrosis in 3, and minimal tumour with substantial necrosis in 12. Autopsy was performed in 2 patients who died 19 and 25 months after implantation. Residual tumour occupied $10 \%$ and $0 \%$ respectively, of the volume of the operative site. The remaining volume was occupied by radionecrotic debris. Further evaluation of this technique in a prospective, controlled setting is warranted.

\section{2.}

\section{Photodynamic Therapy of Brain Tumours: Results in 50 Cases}

\section{P. MULLER and B. WILSON (Hamilton, Ontario)}

We are reporting our experience with intraoperative PDT in 50 patients with malignant supratentorial tumours; in 33 cases the tumour was recurrent. In 45 patients the tumour was a cerebral glioma and in 5 cases a solitary cerebral metastasis. There were 29 males and 17 females with an age range of 17-73 [mean-48] years. Patients received either hematoporphyrin derivative [HPD] or dihematoporphyrin ether [DHE] 18-24 hours pre-operatively. A photo-illuminating device, of the authors' design, was coupled to an argon dye pump laser in order to deliver light at $630 \mathrm{~nm}$ to a tumour cavity created by radical tumour resection and/or tumour cyst drainage. The total light energy delivered ranged from 440 to 3888 Joules and the light energy density ranged from 8 to $175 \mathrm{~J} / \mathrm{cm}^{2}$. In 8 patients a line fiber(s) was used to administer interstitial light as a supplement to the cavitary photo-illumination. The additional light dose range from $60-945 \mathrm{~J} / \mathrm{cm}$.

There were 2 post-operative deaths as the consequence of hematoma accumulation in the tumour resection cavity and in 3 patients neurological function was worse post-operatively and did not recover. There were no adverse systemic reactions to either photosensitizer.

In the group of 45 patients with gliomas the death rate per observation year was 0.92 for the interval between PDT and death. In the interval between first diagnosis and death the rate was 0.41 deaths per observation year. The median survival was 8.6 months with a 1 and 2 year actuarial survival rate of $32 \%$ and $18 \%$, respectively.

In 12 patients a complete or near complete CT scan response was identified post PDT. These patients tended to have a tumour geometry [eg. cystic] that allowed complete or near complete light distribution to the tumour. The median survival for this group was 17.1 months with a $I$ and 2 year actuarial survival of $62 \%$ and $38 \%$, respectively. In the 33 cases who did not have a complete response the median survival was 6.5 months with a 1 and 2 year actuarial survival of $22 \%$ and $11 \%$, respectively.

Photodynamic therapy of malignant brain tumour can be carried out with acceptable risk. Good responses appear to be related to adequate light delivery to the tumour.

\section{3.}

Astroglial Growth Factors in Normal Human Brain and Brain Tumours: Comparison with Embryonic Brain

G.K. SZLAPETIS, M.P. RATHBONE, J-K KIM, R.F. DEL MAESTRO, J. GROVES, J. GILBERT, K. EROLA and R. DeVILLIERS (Hamilton and London, Ontario)

A number of growth-promoting factors from chick brain, such as fibroblast growth factor (FGF), platelet-derived growth factor (PDGF), epidermal growth factor (EGF), Interleukin-1 (Il-1), and glial proliferation factors (GPF) 2 and 4 can stimulate proliferation of the serumstarved cells. Growth factors may play a role in human brain tumours. The activity of a PDGF-like product of the sis oncogene is increased in human glioblastoma multiforme tumours. Moreover, the number of truncated EGF receptors and the expression of EGF receptor gene are enhanced in human malignant astrocytomas and glioblastoma multiforme.

We separated low molecular weight growth factors $(<20 \mathrm{kD})$ from embryonic chick brains using molecular seive chromatography (BioGel P-10). The growth factor activity was assigned to those protein fractions which were able to stimulate proliferation of serum-starved chick brain astrocytes.

From homogenates of 18-day embryonic chick brains and 14-day adult rat brains, normal adult human brain and the human brain tumours, we reproducibly separated 8 fractions that stimulated astrocyte proliferation. One, with an apparent molecular weight $(\mathrm{MW})$ of $9 \mathrm{kD}$, was present in adult human brain, malignant astroglial and oligodendroglial brain tumours and meningiomas.

Growth factor activities were characteristically higher in embryonic than in adult brain. In embryonic brains there was an abundance of lower MW fractions with MW $5 \mathrm{kD}$ to $1.2 \mathrm{kD}$. In adult rat and chick brains, the predominant factors had apparent MWs of 24, 17 and $12 \mathrm{kD}$. In normal adult human brain, the $5 \mathrm{kD}$ factor was present in small amounts and the lower molecular weight factors were present only in very small amounts. In human glioblastoma multiforme (GBM) the predominant activities had apparent MWs of 12 and 5kD with lesser elevations in the 9,2.8 and 1.4kD fractions. In obligodendrogliomas the $9 \mathrm{kD}$ factor was more prominent than in GBM. The growth factor profile of meningiomas was quite different from that of either adult brain or glial brain tumours. Its preponderant activity had an apparent molecular weight of $12 \mathrm{kD}$.

The activity of all factors was decreased by protease treatment of the brain extracts. However, the 5 and $1.2 \mathrm{kD}$ forms were less proteasesensitive than the others. Our data indicate that the factors are probably not derived from high molecular weight factors through protease activity. They also appear to act directly on astrocytes.

4.

Acute Bioenergetic Changes in Malignant Gliomas after Treatment

J.M ROSENTAL, R.L. LEVINE and R.J. NICKLES (Madison, U.S.A.)

Sequential positron emission tomographic scans with [ $\left.{ }^{18 F}\right]-2-$ fluorodeoxyglucose (PET-FDG) were obtained on 14 patients with malignant gliomas. All patients had previously been treated with whole-brain irra- 
diation. Five experimental subjects received adjuvant chemotherapy and 9 control subjects did not. Ratios were determined between the maximal tumour rCMRGlu within a $1 \mathrm{~cm}^{3}$ region of interest (ROI) and the contralateral white matter rCMRGlu within a similar $1 \mathrm{~cm}^{3} \mathrm{ROI}$; this was the glucose uptake ratio. Percent changes in the ratio $I$ day after chemotherapy in experimental subjects, and 30 days after the baseline in controls, were of prognostic significance $(p=0.029)$. Patients whose ratio increased $35 \%$ or more over baseline survived less than 14 months; those with smaller changes survived more than 14 months. The percent change in glucose uptake 1 day after chemotherapy was linearly related to patient survival ( $p>0.005$ ). Both the percent change in glucose uptake and the interval from date of diagnosis were significant covariates in calculating the estimated survival probability.

\section{5.}

Purine Nucleosides and Nucleotides Stimulate Proliferation of Serum Starved Astrocytes in Tissue Culture

\section{M.P. RATHBONE, P.J. MIDDLEMISS and J-K KIM, (Hamilton, Ontario)}

In August 1987, we were purifying, from brain, peptides that stimulated cell division when added to the culture medium of serum-deprived astrocytes. One highly mitogenic fraction, which we had purified to homogeneity in milligram quantities, proved - to our surprise - to contain no amino acids. Analysis of the active material by multiple physicalchemical techniques proved it was guanosine 5' monophosphate (GMP).

A literature survey failed to find any reference to mitogenic properties of GMP. In the belief that mitogenicity of such a well known and ubiquitous compound could hardly have remained undiscovered, we assumed that the active mitogen was probably a peptide present in trace amounts and hence not detected, co-purified with GMP. So convinced were we that GMP was not a mitogen that we set out to prove that it was not. We found, however, that authentic GMP (obtained commercially) also stimulated the growth of astrocytes in the same dose-dependent manner, as did the material purified from brain.

We then identified another mitogenic low molecular weight fraction in the brain extracts as AMP. We therefore questioned which purine nucleosides and nucleotides stimulated astrocyte proliferation and to what extent. In addition to GMP and AMP, guanosine, and adenosine were active in picomolar concentrations and also showed a lesser peak of activity in the micromolar range. The nucleotides were active in the micromolar but not the picomolar range. Surprisingly, the guaninebased compounds stimulated proliferation to a greater extent than the adenine-based analogues.

We investigated the mechanism by which these nucleosides and nucleotides act. They did not act by binding to the surface of the tissue culture dish to modify its surface charge. Similarly, they are active whether or not the culture dish is collagen coated. They do not act by modifying the effects of other growth factors in the culture medium. Their growth-promoting effects on astrocytes are, however, inhibited by low concentrations of theophylline in a dose-dependent fashion, characteristic of phenomena mediated through purine receptors. These data led us to propose the hypothesis that adenine and guanine purine nucleotides and nucleosides stimulate astrocyte proliferation by acting extracellulary through stimulation of purinergic receptors on the surface of astrocytes.

\section{6.}

Stimulation of Astrocyte and Astrocytoma Cell Proliferation by Purine Nucleosides and Nucleotides: Receptors, Mechanisms and Effects of Aging

P.J. MIDDLEMISS, M.P. RATHBONE, G.K. SZLAPETIS and E. HOOFTMAN (Hamilton, Ontario)

As described in a companion paper, low concentrations of purine nucleosides and nucleotides stimulate proliferation of serum-deprived quiescent astrocytes when added to their culture medium. This effect was inhibited, in a dose-dependent fashion, by low concentrations of theophylline, a purinergic receptor antagonist. We therefore used other specific agonists and antagonists of purinergic receptors to further define the way in which these substances were acting. Some of the effects appear to be mediated through a purinergic $P_{1}$ receptor (that responds predominantly to nucleosides and their monophosphates), $A_{2}$ subclass. In addition, however, agonists of $P_{2}$ receptors (that respond predominantly to ADP and ATP) were active in nanomolar ( $P_{2 x}$ agonists) and picomolar $\left(\mathrm{P}_{2 y}\right.$ agonists) concentrations. Some of these agonists were resistant to hydrolysis by ectoenzymes. Therefore it appears that purine nucleotides do not have to be metabolised to nucleosides to stimulate cell proliferation as the non-hydrolyzable nucleotides were active. As nucleotides do not readily cross cell membranes, this also implies that these compounds were acting through receptors on the cell surface.

Stimulation of $P_{1}-A_{2}$ receptors is associated with increased intracellular cAMP levels. Therefore we tested whether the various nucleosides and nucleotides that stimulated cell proliferation also increased intracellular cAMP. Our results indicated that stimulation of cell proliferation by guanosine or by adenosine may be mediated through rises in intracellular cAMP. In contrast to the nucleosides, the purine nucleotides did not significantly elevate intracellular cAMP.

We tested whether cells other than chick brain astrocytes were stimulated by purine nucleosides and nucleotides. Swiss mouse 3T3 cells, chick meningeal fibroblasts, chick myoblasts and a human malignant astrocytoma cell line - SKMG-I - were all stimulated. One astrocyte cell line tested - U373 - was not stimulated however.

When cells in the central nervous system become "sick" or die, they release abundant quantities of nucleosides and nucleotides. Hypoxia is a potent stimulus that releases adenosine from cells. Our data indicate that the quantities of purine nucleosides and nucleotides released from cells under these circumstances would be sufficient to cause proliferation of surrounding cells. This phenomenon may, therefore, play a role in the stimulation of cell proliferation in brain tumours in which the centres are hypoxic and often necrotic. Since the stimulation is not celltype specific, purine nucleosides and nucleotides may also stimulate proliferation of endothelial cells and play a role in the stimulation of new vascularization in brain tumours.

\section{7.}

Superoxide Dismutase, Glutathione Peroxidase and Catalase Activity in Murine C6 Astrocytoma

\section{R.F. DEL MAESTRO, W. MCDONALD and E. STROUDE (London, Ontario)}

The activities of copper-and zinc-containing superoxide dismutase (CuZnSOD), manganese-containing superoxide dismutase (MnSOD), glutathione peroxidase (GSH-Px) and catalase (CAT) have been assessed in murine $\mathrm{C} 6$ astrocytoma growing in monolayer and spheroid culture in vitro and in a number of different anatomical sites in vivo. The most consistent feature of $\mathrm{C} 6$ astrocytoma when compared to normal adult rat cortex was an increase in CAT and CSH-Px activity which was maintained in all the different microenvironments studied. The increased CAT and GSH-Px measured was localized to both the mitochondrial and the non-mitochondrial non-nuclear cellular fraction. The CuZnSOD content of $\mathrm{C6}$ astrocytoma growing in monolayer culture decreased when these cells were grown as spheroids or inoculated in vivo while MnSOD activity remained stable. The in vitro and in vivo growth of $\mathrm{C} 6$ astrocytoma is associated with distinct patterns of cellular and subcellular content of the scavenging enzymes studied which are different from the patterns seen in rat cerebral cortex and are variable depending on the environment in which the cells are grown. The pattern of enzyme expression seen may be modulated by a variety of interacting mechanisms present in distinct microenvironments including the intracellular rates of substrate generation, nutritional availability of constituent metals and concentration of specific lymphokines and other immunoregulating compounds.

Supported by: The Brain Research Foundation 
The Cycling Pool of Cells within Human Brain Tumours: In Situ Cytokinetics using the Monoclonal Antibody Ki-67

\section{S. BREM, A.M.C. TSANACLIS, F. ROBERT and J. MICHAUD (Montreal, Quebec)}

Brain tumour growth results from the relative proportion of cells contained in three populations: a) cycling/proliferative; b) quiescent $\left(\mathrm{G}_{0}\right) / \mathrm{static}$, and c) terminally differentiated/dying. The cycling compartment can be detected by the mouse monoclonal Ki-67 antibody, an available, rapid, safe, sensitive, and specific method for immunostaining of proliferative cells. We report the Ki-67 labelling index (LI) in 48 brain tumours. Malignant brain tumours have elevated Lls, ranging from $6.0 \%$ to $56.9 \%$ : anaplastic astrocytoma, $8.0 \pm 5.97$; glioblastoma multiforme, $10.1 \pm 4.00$; germinoma, 11.7 ; medulloblastoma, $13.1 \pm 6.07$; metastases, $40.3 \pm 11.95$. By contrast, slow growing tumours showed values close to $1 \%$ : acoustic schwannoma, $0.4 \pm 5.1$; pituitary adenoma, $1.0 \pm 1.18$; meningioma, $1.2 \pm 1.65$; low-grade astrocytoma, < 1; pilocytic astrocytoma, 5.6. Human brain tumours can therefore be ranked according to the percentage of cycling cells with the acoustic schwannoma among the least proliferative and the metastatic carcinoma among the most proliferative. Within a given histiotype, the $\mathrm{Ki}-67 \mathrm{Li}$ may have prognostic and therapeutic implications for the individual patient. Already important for neuro-oncology research, the Ki-67 labelling index should be added to the armamentarium of the clinical neuropathologist to complement the standard histopathologic diagnosis with a cytokinetic analysis of cellular proliferation.

\section{9.}

VX-2 Brain Tumour and Normal Brain Concentrations of the NonLipophilic Drug Doxorubicin During Intraarterial Infusions in Rabbits

S.J. HASSENBUSCH, D.M. WHITING, J.H. ANDERSON, M. ISRAEL and T.W. SWEATMAN (Cleveland, Baltimore, and Memphis, U.S.A.)

Conventional brain tumour therapy using lipophilic agents has shown limited effectiveness. Brain distributions of the non-lipophilic agent doxorubicin (Adriamycin, ADR) after intraarterial (IA) infusions were determined in rabbit VX-2 brain tumour model. With right carotid infusions of ADR, concentrations in tumour center (right hemisphere) averaged $3.3 \pm 1.42 \mathrm{microgram} / \mathrm{g}$ (mean $\pm 1 \mathrm{SE}$ ), concentrations markedly higher than in vitro inhibitory concentrations $\left(\mathrm{IC}_{50}\right)$ of doxorubicin for human gliomas. ADR concentrations in brain-aroundtumour (BAT) averaged $2.43 \pm 0.81 \mathrm{microgram} / \mathrm{g}$ and only $0.104 \pm 0.019$ $\mathrm{microgram} / \mathrm{g}$ in left (non-tumour-bearing) hemisphere. In comparison to similar right carotid infusions of 5-fluorouracil (5-FU), ADR concentrations were $70-80 \%$ of 5 -FU concentrations in tumour center and BAT, but only $18 \%$ in left (non-tumour-bearing) hemisphere. In rabbits with VX-2 brain tumours, carotid artery infusions of ADR increased mean survival times by $20.9 \% \pm 8.3$. Femoral artery infusions of ADR caused diameters of VX-2 leg tumours to increase by only $22.4 \% \pm 30.7$ as compared to $126.5 \% \pm 37.7$ and $181.8 \% \pm 24.3$ with intravenous infusions and no infusions, respectively. These findings are supported by a previous animal study using IA infusions of ADR after hyperosmotic blood-brain barrier disruption and a human study using IA infusions of ADR in brain tumour patients. Significant amounts of non-lipophilic drugs, with IA infusions can penetrate areas of brain tumour but still protect normal areas from toxic drug exposure. Coupled with parallel animal survival studies, this suggests IA infusions of non-lipophilic drugs can be effective in minimal side effects in brain tumour therapy.
10.

General Protease and Collagenase (IV) Activity in C6 Astrocytoma Cells, C6 Spheroids and Implanted C6 Spheroids

I.S. VAITHILINGAM, E.C. STROUDE, W. McDONALD and R.F. DEL MAESTRO (London, Ontario)

Tumour growth is dependent on the ability of neoplastic cells to induce angiogenesis. Remodelling of blood vessels requires reconstruction of the collagen (type IV) and non-fibrous protein components of basement membrane which may alter microvessel protein flux. This study assessed the general protease and collagenase (IV) activities of C6 astrocytoma cells in monolayer and spheroid culture and C6 astrocytoma spheroids growing in vivo.

Extracellular release of non-specific proteases and collagenase IV was maximal during exponential cell growth. Increase spheroid size resulted in enhanced extracellular activity of both enzyme groups assessed. The size of the implanted spheroid influenced the activity measured in vivo. General proteolytic activity was significantly greater in tumour tissue at all spheroid sizes implanted when compared to contralateral cortex while only the implantation of 750 um spheroids resulted in significantly increased collagenase type IV activity.

The growth of C6 Astrocytoma cells in monolayer and spheroid culture in vitro and in vivo is associated with distinct alterations in intracellular and extracellular activity of the proteolytic enzymes assessed. Increased extracellular release of these enzymes may play important roles in tumour angiogenesis, tumour invasiveness and tumour-associated edema.

Supported by: The Brain Research Fund Foundation

11.

Spatially Localized 1H Magnetic Resonance Spectroscopy of an Intracerebral Glioma in the Rat

B.D. ROSS, H. MERKLE, K. HENDRICH, R.S. STAEWEN and $M$. GARWOOD (Minneapolis, U.S.A.)

The need for an improved understanding of the biochemistry and pathophysiology of malignant brain tumours is indicated by the limited success of present therapeutic modalities. Because rodents are most commonly used as models in brain tumour research, it is important to employ a sensitive MRS method which can reproducibly localize spectra to small volumes located anywhere in the brain. Here we present a sensitive ${ }^{1} \mathrm{H}$ MRS localization method to non-invasively map metabolites through rat brains harboring malignant gliomas.

Intracerebral gliomas were induced in rats by injection of $1 \times 10^{6} \mathrm{C} 6$ glioma cells in the frontal lobe 16-20 days prior to MR experiments. A $16 \mathrm{~mm}$ diameter surface coil was placed over the rat head for MRI and iH MRS experiments. Surface coil MRI was performed to determine the size and location of the tumour to obtain optimal parameters for spatially localized 'H MRS experiments.

Spatial localization was accomplished with a hybrid approach based upon ISIS localization in two dimensions (to select a column) and spectroscopic imaging in a third dimension (along the axis of the ISIS column). 'H MRS sensitivity was optimized by using a surface coil transmitter/receiver in conjunction with a modified adiabatic plane rotation pulse (BIR-4) capable of executing semi-selective excitations necessary for water suppression.

Spatially resolved ' $H$ spectra of intracerebral glioma $(n=5)$ revealed significantly decreased concentrations of $\mathrm{N}$-acetyl aspartate and creatine and increased levels of choline compounds and lactic acid (or lipids) as compared to the contralateral hemisphere.

This IH MRS method is capable of monitoring the metabolism of brain tumours from extremely small volumes of tissue (e.g., 28ul) and our results demonstrate that significant metabolic abnormalities associated with intracranial gliomas can be resolved. From future localized ${ }^{1} \mathrm{H}$ 
MRS studies of intracerebral tumours in rats, greater understanding may be gained which will improve the ability to diagnose and treat malignancies in humans.

12.

MRI/MRS Studies of the Effect of Therapy on Malignant Gliomas in Rat Brains

D. WILKINS, P. RAAPHORST, G.R. SUTHERLAND, I.C.P. SMITH and J.K. SAUNDERS (Ottawa, Ontario and Winnipeg, Manitoba)

Malignant gliomas are relatively resistant to radiation therapy since they have shown significant capacity for repair of radiation damage. We have studied an animal model, 9L gliomas in rat brains, in order to explore the effectiveness of other therapies in conjunction with radiation treatment. The techniques chosen to evaluate the various therapies were a combination of magnetic resonance imaging (MRI) and spectroscopy (MRS). MR imaging permits non-invasive measurement of tumour size and provides information on tumour morphology. MRS permits the evaluation of energy status, $\mathrm{pH}$ and the determinations of the concentrations of a number of metabolites, including lactate and $\mathrm{N}$-acetyl aspartate.

The first experiments verified that MRI can be used to define tumour volume in this animal model. A number of rats were implanted with the 9L glioma cells and tumour progression monitored by MRI. Excellent correlation was observed between MRI and histopathological determinations of tumour volume. This was valid for both irradiated (up to 90 Grey of Cobalt-60) and non-irradiated tumours. MRI and histopathology also confirmed the radio-resistant nature of this line of gliomas. Changes in MRI parameters of tumour tissue post-irradiation was observed in about $30 \%$ of the rats.

Since tumour diameter is of the order of $3-5 \mathrm{~mm}$, localized $31 \mathrm{P}$ spectra only from the tumour is very difficult. We have used ISIS to obtain both $\mathrm{IH}$ and $31 \mathrm{P}$ spectra from tissue which is predominantly but not exclusively tumour tissue. The presentation will include a discussion on the validity of localization techniques as well as the results obtained in this study.

13.

Glucose Phosphate Isomerase as a CSF Marker for Leptomeningeal Metastasis

H.B. NEWTON, M. FLEISHER and M.G. MALKIN (New York, U.S.A.)

Glucose phosphate isomerase (GPI), also known as phosphohexose isomerase, is a glycolytic enzyme whose activity is elevated in serum of patients with primary and metastatic nervous system tumours. To improve the diagnostic accuracy of leptomeningeal metastasis (LM) we measured GPI levels in cerebrospinal fluid (CSF) of 66 patients with both central nervous system and systemic malignancies. GPI was determined kinetically using a coupled enzyme reaction assay. There were 31 males and 35 females, age 1-76. Thirty-one had primary brain tumours, and 35 systemic cancer with suspected CNS metastasis. Ninety-five samples were analyzed; GPI values ranged from 0.85 to $329.0 \mathrm{U} / \mathrm{L}$. Compared to positive CSF cytology and myelography, GPI sensitivity was $53.5 \%$ and specificity $92.1 \%$ for the group as a whole. There was a highly significant association between elevated CSF GPI ( $>20 \mathrm{U} / \mathrm{L})$ and LM $\left(X^{2}=17.12, p>0.001\right)$. Results were similar when primary CNS and systemic malignancies were considered separately. One patient with primary CNS lymphoma and initially elevated CSF GPI, sampled serially, showed progressive clinical improvement as GPI levels normalized with administration of intrathecal methotrexate. Although not very sensitive, demonstration of elevated CSF GPI presents strong evidence of LM and may permit earlier diagnosis of this serious complication of cancer.
14.

Giant Demyelinating Plaques Creating the Impression of Brain Tumours. Multiple Sclerosis or Post-Infectious Encephalitis? A Review of 30 Cases

\section{J.J. KEPES (Kansas City, U.S.A.)}

During the last decade Neurosurgeons, Neuroradiologists and Pathologists have encountered large demyelinating lesions in the brain (some of them causing significant mass effect) that appeared as neoplasms radiologically, but were identified as demyelination on biopsy. Uni-and multifocal forms exist, in the latter forms the lesions are of the same age. Most cases present as deep hemispheric white matter lesions without predilection to periventricular areas, optic system, cerebellum or brain stem. Corticosteroid treatment appears very effective and impressive improvement on computerized tomographic (CT) scans can be seen on follow-up studies. Perhaps the most striking feature of the cases we had the opportunity to observe, was the lack of exacerbations after the initial lesion or lesions subsided on treatment. The longest postoperative survival observed is 19 years, but recurrences were not observed in that or our other patients with shorter follow-up periods. Since similar lesions have been observed by others following inoculation with the swine-flu vaccine it is possible to contemplate that postinfectious/postvaccination encephalitis is not restricted to the usual form with tiny perivenous foci of microscopic demyelination. but may at times present with very large focal lesions. The distinction from classical multiple sclerosis appears very important when contemplating prognostic outlook for the patient.

\section{5.}

In Vivo Measurement of Microvascular Permeability in Brain Tumours

T-Y LEE, W-T YEUNG, R.F. DEL MAESTRO, T. BROWN and R. KOZAK (London, Ontario)

The leakiness (permeability) of brain capillaries is related to two important problems in brain tumour research, namely, (i) the formation of brain edema and (ii) delivery of anticancer drugs. Measurement of microvascular permeability (PS) could result in better steroid and anticancer drug therapy in brain tumours.

We have developed an in vivo method of measuring PS in brain tumours using Isovue (lopamidol) as the contrast agent and a clinical $X$-ray CT scanner. In patient studies, the position of the tumour was located by an initial CT scan of the head. Next two baseline scans at the chosen position and a background arterial sample from a radial artery were obtained. Sampling of arterial blood and CT scanning of the tumour site were repeated at different times up to $48 \mathrm{~min}$ after injection of Isovue 300 (@1 ml/Kg body weight). After the study, the blood samples were centrifuged to obtain plasma samples which were then scanned in a brain phantom to measure the concentration of Iopamidol in plasma in units of CT number increases over the background plasma sample. Similarly the tissue concentration of Iopamidol was determined by subtraction of the baseline scan from the enhanced CT scans. The leakage of lopamidol into the brain through the blood-brain-barrier is modelled as an exchange process between two compartments, the tissue plasma space and the tissue interstitial space. Using this model and the concentration measurements in blood and tissue, quantitative estimated of microvascular permeability (PS) and plasma volume $\left(V_{b}\right)$ in the CT slice containing the tumour were calculated and displayed as a pseudocolour image overlaid on the conventional CT scan.

In a preliminary study of ten patients with Anaplastic Astrocytoma (AA) $(n=2)$, Glioblastoma Multiforme $(G M)(n=3)$ or Metastases (MA) $(n=5)$, the functional images showed variations in PS and $V_{b}$ within the tumour which were difficult to perceive in the original enhanced CT scans. Although there was a general trend of increase in 
PS and $V_{b}$ in the progression from AA to MA, the differences in PS values among the three groups were not signifcant and only the $V_{b}$ values for the AA and MA groups were significantly different. We have attributed the wide spread in the measured values to the effect of steroids which these patients had been prescribed for variable periods of time before the study.

16.

The Effect of Radiation on Normal Human Brain Assessed by Nuclear Magnetic Resonance Spectroscopy

S.K. ALBO, R.C. URTASUN, P.S. ALLEN, D. HUYSER-WIERENGA and D. FULTON (Edmonton, Alberta)

We are involved in a prospective non-invasive study to assess late radiation effects in the central nervous system of humans following high doses of therapeutic radiation,. Relative cerebral metabolite concentrations are determined through comparison of the areas of peaks visualized through NMR spectroscopy. Statistical analysis of these peak ratios from phosphorous spectroscopy indicates no significant changes occurring in phosphorus-containing cerebral metabolites following radiotherapy. In the case of the proton spectroscopy, preliminary results suggest that levels of choline and $\mathrm{N}$-acetyl aspartate vary substantially between irradiated and non-irradiated brain.

Methods: At present 20 patients are entered in this study. Thirteen are being analyzed using phosphorus spectroscopy while the other seven are involved in a proton spectroscopy study. These patients have received up to $8000 \mathrm{cGy}$ of high energy $x$-rays. The patients are analyzed prior to radiotherapy, then at intervals of $0,2,4,8$ and 12 months following RT. During each NMR session, spectra are acquired from a region of brain receiving high doses of radiation (80-90\% of total), and another region which receives lower doses (approximately 50\% of total). A group of normal volunteers has also been examined.

Results: 1) Phosphorus spectroscopy may not be sensitive to radiation-induced changes in cerebral metabolites. 2) The steady decline of $\mathrm{N}$-acetyl aspartate (NAA) seen in irradiated normal brain suggests that brain tissue is affected by therapeutic doses of radiation. Both the high dose and low dose regions of brain exhibit this metabolic change although higher doses seem to cause greater changes.

17.

Effect of Radiation on Rat Brain Tissue by Magnetic Resonance Imaging

D. WILKINS, P. RAAPHORST, I.C.P. SMITH, G.R. SUTHERLAND, and J.K. SAUNDERS (Ottawa, Ontario and Winnipeg, Manitoba)

Malignant gliomas are highly radiation resistant, and treatment doses are limited by the radiation response of surrounding normal brain tissue. Many glioma patients experience neurological deficit attributable to the effects of radiation therapy. Detection of radiation-induced injury and correlation with CNS changes is important for understanding and possibly treating or preventing these adverse effects of demyelination, capillary damage, blood brain barrier opening and glial and neuronal necrosis, which may be detectable by magnetic resonance imaging.

In this study, normal Fischer rats underwent cobalt -60 radiation therapy to a localized region of the brain. Single doses of 60 to 80 Gy were used, with a beam width of $5.2 \mathrm{~mm}$. MR imaging showed no evidence of radiation damage to normal brain on $T 2$ weighted images up to five weeks following irradiation. However, rats inoculated with $9 \mathrm{~L}$ gliomas and irradiated showed increased signal attributable to radiation damage in both tumour and surrounding normal brain within 1 week of treatment. This indicates that normal brain may be more susceptible to radiation damage when a tumour is present. Ongoing imaging studies, correlated with histological analysis, are using MRI to further investigate detection of long-term radiation injury to normal brain, with and without intracranial tumours present.
18.

Malignant Glioma - Patterns of Recurrence Following Whole Brain Plus Boost Radiotherapy

L.E. GASPAR, B. FISHER, D.R. MACDONALD, D. LEBER, S.C. SCHOLD and J.G. CAIRNCROSS (London, Ontario and Durham, U.S.A.)

We conducted a retrospective study of 70 patients with malignant glioma (48 glioblastoma multiforme, 22 anaplastic astrocytoma) treated on a Phase III study. All received radiotherapy (RT) ( $44 \mathrm{~Gy} / 22$ fractions/4.5 weeks whole brain, $14 \mathrm{~Gy} / 8$ fractions/1.5 weeks tumour boost) followed eight weeks later by chemotherapy. The boost was usually planned using the post-operative or a mid-RT planning CT scan, not the pre-operative CT. We analyzed simulator films and CT scans prior to surgery and at recurrence to determine the adequacy of the boost (i.e., location and size of minimum margin) and sites of recurrence in relation to the boost. The original tumour was entirely within the boost (positive margin) in $42(60 \%)$, at the edge of the boost (no margin) in $16(23 \%)$, and partly outside the boost (negative margin) in $12(17 \%)$. The tumour recurred in 53 patients and was in the boost in $37(70 \%)$, partly outside the boost in $9(17 \%)$, out of the boost (within brain) in $5(9 \%)$, in the boost and spinal cord in $1(2 \%)$ and in the spinal cord only in $1(2 \%)$. We then analyzed the 14 patients in whom the recurrent intracerebral tumour was entirely or partly outside the boost. In 10/14 the tumour recurred at the minimum margin and in $7 / 14$ the boost had missed part of the tumour. The pattern of recurrence did not affect survival in this small series. Most tumours recurred at the original site and were within the boost but the tumour recurred partially or entirely outside the boost in $7 / 12$ patients with negative margins. Now that we have eliminated whole brain radiation treatment and use only limited fields, we recommend generous volumes designed using the pre-operative $\mathrm{CT}$ scan.

\section{9.}

A Phase I RTOG Study of Iododeoxyuridine (IUdR) and Radiotherapy in the Treatment of Primary Malignant Brain Tumour

D.F. FULTON, R.C. URTASUN, S.G. LESTER, M. SILVERSTEIN, T. WASSERMAN, K. MARTZ and S. McKINNON) Philadelphia, U.S.A.)

IUdR substitutes for thymine in DNA of rapidly dividing tumour cells. IUdR administration may enhance tumour cell kill by radiation without change in normal tissue toxicity. Patients with proven malignant glioma are eligible for this phase I trial, (RTOG-86-12) searching for the optimal dose and scheduling of intravenous IUdR in combination with radiation. The tumour radiation dose is $60 \mathrm{~Gy}$ in 32 fractions in six and a half weeks. In the first step, the dose of IUdR was $1 \mathrm{~g} / \mathrm{m}^{2}$ daily, by continuous intravenous infusions for 96 hours each week for six weeks. 22 patients were entered and are evaluable and 20 completed radiation as per protocol. 11 patients received at least $20 \mathrm{~g} / \mathrm{m}^{2} \mathrm{IUdR}$. The drug was stopped in 2 patients due to hematological toxicity, 6 due to liver, and 2 due to skin, 1 due to GI toxicity and 1 patient developed herpes esophagitis. The median survival was 58 weeks. 15 patients had hematologic toxicity ( 2 grade 3 ), 12 patients had GI toxicity and 10 patients had liver enzyme changes which were reversible in some patients despite continued drug administration without dose modification. 6 patients had a maculopapular rash and 1 had Stevens-Johnson syndrome. 5 of these, including the one with Stevens-Johnson syndrome, were taking Diphenelhydantoin. 10 patients had ridging and softening of the nails. In the second step, the dose of IUdR was $2 \mathrm{~g} / \mathrm{m}^{2}$ daily, by continuous intravenous infusion for 48 hours each week for 6 weeks. So far, 9 patients have been entered and 5 completed radiation as per protocol. One patient died prior to starting any treatment and the other 3 are currently beginning treatment. Four patients received at least $20 \mathrm{~g} / \mathrm{m}^{2}$ IUdR. The drug was stopped in 1 patient due to hematological toxicity plus liver enzyme changes, 1 due to GI toxicity and 1 due to skin toxicity and 
stomatitis. Of the 5 patients who completed treatment, 1 had hematological and 4 GI toxicity. I had liver enzyme changes, 4 had stomatitis. I patient, who was also taking Diphenylhydantoin, had a skin rash. 2 patients had ridging and softening of the nails.

The incorporation of IUdR into tumour tissue obtained at surgery is also being assessed. One patient studied was given IUdR $1 \mathrm{~g} / \mathrm{m}^{2}$ by continuous infusion for 24 hours prior to tumour resection. IUdR, measured by monoclonal antibody technique, was present in $14 \%$ of brain tumour cells.

20.

Radiation Time Dose Fractionation Studies (Hyperfractionation) in Malignant Glioma

R. URTASUN, D. FULTON, H. THOMAS, I. SCOTT-BROWN, D. HUYSER-WIERENGA and C. FLOOD (Edmonton and Calgary, Alberta)

Clinical experience has shown that human malignant gliomas have a dose response curve up to $6000 \mathrm{cGy}$. Increasing the radiation dose beyond this level might result in better tumour control and patient survival but might also increase the incidence of delayed normal tissue injury. We postulate that altering the dose of radiation per fraction, the number of fractions and their timing might allow the delivery of higher total doses of radiation to the brain without increasing toxicity. We treated a total of 278 patients with supratentorial malignant glioma with $6141 \mathrm{cGy}, 7120 \mathrm{cGy}$ or $8000 \mathrm{cGy}$, utilizing 3 daily fractions separated by 4 to 5 hour intervals in an overall time of 5 and a half weeks. There was a significant improvement in median time to tumour progression and median survival in patients treated with $8000 \mathrm{cGy}$ as compared to those treated with $7120 \mathrm{cGy}$. However, this statistical significance was lost when results were adjusted for the known prognostic variables of age, histological grade and Karnofsky performance status. So far, there is a strong trend toward prolonged time to tumour progression and median survival in the subgroup of patients younger than age 46 with glioblastoma multiforme treated with $8000 \mathrm{cGy}$ compared to similar patients treated with $7120 \mathrm{cGy}$. We have observed a dose response curve from 6000 to $8000 \mathrm{cGy}$ for acutely reacting tissues such as the skin. However, we have not yet seen an increase in CNS late effects by increasing the dose beyond $6000 \mathrm{cGy}$.

21.

Iodine-125 Temporary Interstitial Implants for Recurrent Malignant Astrocytomas of the Brain

\section{N.J. LAPERRIERE, M. BERNSTEIN, P.M.K. LEUNG, S. MCKENZIE} and M LUMLEY (Toronto, Ontario)

We report our experience with interstitial brachytherapy in 25 consecutive patients with recurrent malignant astrocytomas of brain. All implants were performed stereotactically with the temporary placement of linear arrays of high activity iodine- 125 seeds utilizing the BrownRoberts-Wells stereotactic head frame. There were 17 males and 8 females. Age ranged from 16 to 73 years with a median of 47 . Histologically, there were 21 patients with glioblastoma multiforme and 4 patients with anaplastic astrocytoma. The tumours were recurrent in 23 patients, and in 2 patients the tumours developed 9 and 22 years after prior external radiotherapy for a pituitary adenoma and an optic glioma respectively. In the 23 patients with recurrent tumours, the interval from their initial surgery to brachytherapy ranged from 26-156 weeks, with a median of 47 weeks. Brachytherapy was the only treatment in 24 patients, while 1 patient had chemotherapy prior to and after the implant as additive therapy. Follow-up from the time of implant ranges from 4176 weeks, with a median of 76 weeks. Median survival for all patients from time of implant calculated by the Kaplan-Meier method is 54 weeks. Thus far in the follow-up of these patients, $8 / 25(32 \%)$ have undergone reoperation for intractable mass effect. Time to reoperation post-implant ranges from 11-106 weeks with a median of 28 weeks. The median survival of the 8 patients undergoing reoperation post-implant is 54 weeks. We conclude that brachytherapy is of benefit in prolonging survival in selected patients with recurrent malignant astrocytoma of brain.

22.

The Value of Reoperation for Recurrent Malignant Astrocytoma

M. BERNSTEIN, P. DIRKS, P. MULLER and W. TUCKER (Toronto, Ontario)

The authors conducted a retrospective review to re-examine the value of second operation for patients with malignant astrocytoma. The experience of three University of Toronto Neurosurgeons was collected. Eligible patients were those who were reoperated for recurrence of a previously treated malignant astrocytoma (ie. grade III and IV astrocytomas). Patients who were reoperated more than once and those receiving any form of experimental therapy after the second craniotomy were excluded from study.

The study population comprised 42 patients of median age 51.5 years. There were 26 males and 16 females. Ten were originally treated for a grade III astrocytoma and 32 for glioblastoma multiforme (ie. grade IV tumour). All patients were originally treated with craniotomy and external radiation $(50 \mathrm{~Gy} / 25$ fractions via parallel opposed regional fields). All patients had Karnofsky performance status of 50 or greater at time of second operation. Median interval between first and second operation was 31 weeks. As of February 1990, 34 patients are dead and 8 remain alive. Median survival following repeat craniotomy calculated by the Kaplan-Meier method is 20 weeks. Complication rate of second craniotomy was $11.9 \%$ (there were 3 brain abscesses and 2 infected scalp flaps).

Based on this limited review we conclude that second craniotony alone for recurrent malignant astrocytoma confers a modest prolongation of survival with reasonable preservation of quality of life. Patients who satisfy the clinical and surgical criteria for second craniotomy for recurrent malignant astrocytoma should as well be offered some form of experimental therapy.

23.

Decompression of Brain Tumour Cysts by Percutaneous Ommaya Reservoir System Aspiration

\section{L.R. ROGERS and G. BARNETT (Cleveland, U.S.A)}

Symptomatic brain tumour cysts (BTC)s pose a difficult management problem, particularly when they are not surgically resectable. The optimal method for BTC decompression has not been defined. We have successfully performed BTC decompression by percutaneous aspiration of cyst fluid via the Ommaya reservoir system (ORS) in 21 symptomatic BTCs in twenty patients. Age ranged 3-70 years, median 50 years. Sixteen were primary tumours (12 anaplastic glioma, 2 craniopharyngioma, 1 oligodendroglioma, 1 brainstem glioma) and four were metastatic. One craniopharyngioma was multi-cystic and 2 ORSs were placed. Locations were cerebral hemisphere (18), suprasellar (2), brainstem (1). Maximal cyst diameter on transaxial CT ranged 2.5-6 centimeters. Fourteen had CT appearance of a true cyst, 7 a pseudocyst. Nineteen catheters were placed through twist drill holes via CT stereotactic guidance; two through burr holes via CT guidance. Cyst fluid was effectively aspirated from 1-15 times in each patient, range of 3-50 cc. Aspiration by ORS was considered ineffective in 1 patient, however, because of rapidly accumulating cyst fluid requiring frequent aspiration and ultimately permanent drainage. Small asymptomatic intratumoural hemorrhage was noted on CT in two patients after placement. No other acute or delayed complications occurred at follow-up of one month to 
$2^{1 / 4}$ years. In our experience BTC aspiration by ORS has been equally effective as burr hole needle aspiration but does not require CT guidance in deep-seated BTCs and requires minimal technical skill. It has been more effective than cystoperitoneal shunting.

\section{4.}

\section{The Laitinen Stereotactic Brain Biopsy Apparatus}

\section{R.G. PERRIN, B. NIXON and A. NORTH (Toronto, Ontario)}

Most stereotactic systems in current use are invasive - requiring pins fixation of the frame to the skull. Consequently, placement of the frame, radiographic imaging studies and surgical biopsy must follow in close sequence to minimize patient discomfort and the risk of dislodging the frame.

The Laitinen method uses a simple CT and MRI compatible apparatus which is easily mounted, removed and remounted with a high degree of accuracy and reproducibility and which allows temporal separation of imaging studies and surgical biopsy.

We have applied the Laitinen method to 35 patients for localization, biopsy and drainage of suspected neoplastic, infective, and haemorrhagic brain lesions. One patient suffered a fatal, massive subarachnoid haemorrhage. Definitive diagnosis was not obtained in seven patients. There were no other complications associated with use of the apparatus.

The Laitinen frame provides a useful adjunct for biopsy and aspiration of brain lesions in selected patients.

\section{5.}

\section{Phase I Study of Intracarotid (IC) Carboplatin}

\section{STEWART, Z. GRAHOVAC, L. GIONET, B. BENOIT and H.} HUGENHOLTZ (Ottawa, Ontario)

11 patients were treated on a phase I study of IC carboplatin 200$400 \mathrm{mg} / \mathrm{m}^{2}$ in D5W infused over $15-30$ minutes through a transfemoral catheter with a 0.2 micron in-line filter. This study was done since IV carboplatin has less retinal and neurotoxicity than cisplatin and is active vs brain tumours. 8 males and 3 females ranging in age from $37-72 \mathrm{yr}$. (median, 59) were treated. ECOG performance status was 1 in 2, 2 in 3, and 3-4 in 6 patients. 7 had had 1-3 prior chemotherapy regimens. 10 had failed prior radiotherapy. Response was: glioblastoma: 4 failed; recurrent grade II glioma: I PR, I failed; malignant oligodendroglioma: 1 failed; brain metastases from nonsmall cell lung cancer: 1 PR, 1 stable, 1 failed. Median survival was 10 weeks. Nausea was mild to moderate. I patient had granulocytopenia and 2 had thrombocytopenia (mild). at $200 \mathrm{mg} / \mathrm{m}^{2}$ (2 patients), 1 had a focal seizure. At $300 \mathrm{mg} / \mathrm{m}^{2}$ ( 7 patients), 2 with abnormal small arteries had severe pain early during treatment and post-treatment ipsilateral conjunctival edema, decreased vision, and cerebral edema (with partially reversible increased hemiparesis), 1 other had mild decrease in ipsilateral vision and 1 had transient aphasia on removal of the catheter (? spasm). at $400 \mathrm{mg} / \mathrm{m}^{2}$ (2 patients), both have had decreased ipsilateral vision. The dose recommended for phase II studies is $300-400 \mathrm{mg} / \mathrm{m}^{2}$. Retinal toxicity is dose-limiting. Treatment should not be given if the carotid is abnormally small and should be stopped immediately if local pain develops.

Supported by: Bristol Pharmaceuticals Canada

\section{6.}

\section{Predicting Good or Bad Outcome in Malignant Glioma}

D.R. MACDONALD, P. BRASHER, B. SMUCK and J.G. CAIRNCROSS (London, Ontario)

We retrospectively reviewed the clinical, pathologic, radiologic and surgical characteristics of 285 consecutive adults with newly diagnosed biopsy-proven supratentorial malignant glioma treated at a regional cancer center from July 1982 through December 1987 , to identify the factors affecting length of survival. Multivariate analysis demonstrated that age, duration of symptoms, preirradiation (post-operative) performance status, tumour histology, accessibility to resection, extent of resection, radiotherapy and prior low-grade glioma were significant independent variables influencing survival (J. Neurosurg 1989; 71: 487-493). We are using this data base, updated for survival and Karnofsky performance status (KPS) at 6 month intervals, to identify those factors present at diagnosis that would reliably predict either good outcome (alive with KPS $\geq 70,1$ year after diagnosis) or bad outcome (dead, or KPS $\leq 50$, 6 months after diagnosis). These predictive formulas will be validated by examining their ability to predict good or bad outcome in all patients treated at our center from January 1988 through December 1989. Such information would be important to support treatment or no treatment decisions in patients with newly diagnosed malignant glioma.

\section{7.}

Mer Phenotype, Glutathione Levels and Inherent Radiosensitivity as Predictors of Tumour Treatment Response in Patients with Malignant Gliomas

\section{R.S. DAY ALLALUNIS-TURNER III and D. HUYSER-WIERENGA (Edmonton, Alberta)}

We postulate that the ability to repair 06-Methylguanine lesions in DNA (Mer Phenotype), Glutathione (GSH) levels and inherent radiosensitivity can be used in conjunction with clinical parameters to predict the resistance of individual patients with malignant glioma to therapy. Tumour biopsy material was obtained from 69 patients. 6 were MER negative. There was a positive correlation between sensitivity to nitrosourea in vitro and Mer negative phenotype in both biopsy material and biopsy derived tumour cell lines. 3 Mer negative patients were treated with nitrosourea chemotherapy. 1 patient treated with CCNU immediately following his initial diagnosis had a partial response after 1 course of therapy. I patient treated with $\mathrm{CCNU}$ at the time of tumour recurrence had stable response to 2 courses of CCNU. I patient treated with high dose BCNU was continuing to show decrease in size of enhancing tumour 22 weeks after treatment when he died of pulmonary complications. Significant variation in tumour GSH content was observed. The average GSH content of biopsy samples was 7 fold lower than that of the corresponding cell lines $\left(0.74\right.$ vs $5.08 \mathrm{mmol} \mathrm{GSH} / 10^{6}$ cells). Attempts to chemosensitize Mer positive tumours by depleting tumour GSH levels were successful in 11 of 22 tumours tested. The inherent radiosensitivity of early passage tumour cell lines was also assessed by determining the surviving fraction (SF) of cells after $2 \mathrm{~Gy}$ radiation (SF2). The majority of tumours were relatively radio-resistant ( $S F 2>0.5$ ); however, 2 radiosensitive tumours were identified $(\mathrm{SF} 2<0.2)$. Times to tumour progresssion after completion of radiation therapy for these two patients were 18 and 58 weeks. These findings demonstrate that patients with malignant glioma can exhibit considerable heterogeneity. Determining the repair potential and chemo/radiosensitivity prior to therapy may help to identify specific treatment sensitive individuals.

\section{8}

\section{Primary CNS Tumours in Children Long Term Survival after} Radiation Treatment

\section{JENKIN and A. VATTER (Toronto, Ontario}

The Toronto data base (1958-85) for children who were irradiated for a primary CNS tumour was examined to determine survival 5-20 years after diagnosis and, where death occurred after 5 years, the cause of death. 
Overall provisional actuarial results were:

\section{SURVIVAL AT YEARS}

\begin{tabular}{lrcccc} 
Tumour Type & Number & $\mathbf{5}$ & $\mathbf{1 0}$ & $\mathbf{1 5}$ & $\mathbf{2 0}$ \\
\hline All tumours & 612 & 70 & 61 & 56 & 52 \\
Astrocytoma - High grade & 79 & 55 & 43 & 33 & 26 \\
Astrocytoma - Low grade & 239 & 77 & 69 & 64 & 61 \\
Ependymoma & 71 & 66 & 49 & 49 & 49 \\
Medulloblastoma & 151 & 74 & 61 & 59 & 52 \\
Spinal Cord & 38 & 89 & 89 & 82 & 82
\end{tabular}

The dominant cause of death up to 15 years from diagnosis remained progression or spread of the primary tumour. Of those patients lost to follow-up, $33 \%$ were lost during the first two years from diagnosis and $50 \%$ during the first five years. Therefore the computation of actuarial survival rates censoring patients lost to follow-up may bias the results favourably. Work is ongoing to update these data. A report will be made on the overall and relative incidence of fatal second tumours and other cause of death.

29.

Spinal Fixation Following Anterior Decompression for Symptomatic Spinal Metastases

A. NORTH, B. NIXON, R.G. PERRIN, R.J. McBROOM (Toronto, Ontario)

Surgical strategies for the treatment of symptomatic spinal metastases (SSM) must allow for both decompression of the spinal cord and nerve roots, and stabilization of the spinal column.

Our experience with anterior decompression and stabilization for SSM includes 59 patients ( 24 male and 35 female). Primary sites of tumour origin were: breast, lung and prostate. Indications for surgical intervention were: local pain, progressive neurologic deficit, and spinal instability. Preoperatively 40 patients were ambulatory, 15 were bed-ridden, and 4 were frankly paraplegic. Surgical intervention involved anterior cervical ( 12 cases) transthoracic ( 37 cases), or transabdominal lumbar (10 cases) exposures with vertebral corpectomy and decompression of the dural sac and the nerve roots. Stabilization was secured with a Ushaped stainless steel plate and interposed methyl methacrylate strut.

Post-operatively 41 patients were ambulatory. 49 were improved and 10 were unchanged or worse.

Complications included: graft dislodgement, wound infection, respiratory failure, esophageal perforation, coagulapathy, dysphagia, and prolonged air leak.

30.

The Prussian Blue Reaction as an Aid to Tumour Diagnosis

K. MAILER and R.F. DEL MAESTRO (Halifax, Nova Scotia and London, Ontario)

The traditional Prussian Blue reaction can be modified to a negative stain for tissue peroxidases. We extended this principle to differentiate tumours (which have high catalase levels) from the normal surrounding tissue (lower catalase levels). We used a $\mathrm{C}_{6}$ astrocytoma rat model and also a variety of human malignancies. Freshly excised tissue slices are applied briefly to a support matrix when treated with hydrogen peroxide and then iron salts reveals a clearly differentiated border between tumour edge and normal brain tissue. This procedure takes two minutes and requires unsophisticated, inexpensive materials. Thus, the technique may find use as an aid in tumour diagnosis during the operating procedure.

\section{1.}

\section{Cerebral Neoplasms Studied Using High Resolution 'H NMR Spectroscopy}

\section{J. PEELING and G.R. SUTHERLAND (Winnipeg, Manitoba)}

Quantitation of metabolites observed in clinical nuclear magnetic resonance (NMR) spectroscopic examinations remains problematical. As the metabolites giving the sharpest peaks in 'H spectra are low molecular weight water soluble species, one approach to this problem is the study by NMR of perchloric acid (PCA) extracts of tissue obtained by surgical biopsy. This avoids problems of localization, produces high resolution spectra which can be correlated to the less well resolved clinical spectra, and gives spectra readily quantitated by the addition of standard compounds. In this study high resolution IH NMR spectroscopy of PCA tissue extracts has been used to quantify the levels of numerous small metabolites [alanine, glutamate, glutamine, gammaaminobutyric acid (gaba), $\mathrm{N}$-acetylaspartate (naa), aspartate, taurine, glycine, acetate, creatine, cholines, myoinositol] in surgical biopsy material obtained from patients with epilepsy and intracranial neoplasms. Tissue was immediately frozen in liquid nitrogen, extracted with PCA, neutralized, and lyophilized. Each sample was dissolved in $D_{2} 0$ containing $0.75 \mathrm{mM}$ TSP as an internal concentration and chemical shift reference. ${ }^{1} \mathrm{H}$ NMR spectra were assigned to comparison with spectra of pure compounds, and quantitated by integration.

Brain tissue from epilepsy patients consisting of mainly (ca. $90 \%$ ) of grey matter was taken to be representative of "normal" brain. Levels of amino acids determined in these samples are in excellent agreement with literature values.

Spectra of extracts of tumours differ markedly from those of "normal" brain. Mannitol, administered perioperatively, is observed only in the spectra of tumour extracts, in keeping with the known endothelial permeability of intracranial neoplasms. The absence of very low levels of naa and gaba in the tumours reflects the absence of neurons, as these metabolites are neuronal in origin. A shift in the glutamate/glutamine ratio towards glutamine may have a similar origin as synthesis of glutamate from glutamine is a neuronal process. Of other changes in metabolite levels, a decrease in total creatine pool in tumours is notable, possibly reflecting the importance of the production of ATP by glycolysis rather than aspiration in brain tumours. The observation of glucose, absent in all "normal" brain extracts, in all tumour extracts, may have a similar origin, as brain tumours have been shown to have a substantially elevated glucose uptake and consumption.

Although the spectra of all tumour extracts are highly variable, some significant differences are evident between tumour types. Meningiomas are regionally quite uniform, while (malignant) astrocytomas show substantial regional variability, with levels of all metabolites decreasing towards the center of the neoplasm, corresponding to pathologically defined necrosis. Meningiomas are further distinguished from astroyctomas by the virtual absence of creatine compounds in meningiomas.

32.

\section{Incidence of Clinicopathological Features of Meningioma}

\section{LOUW, G.R. SUTHERLAND, M. ROHRINGER, A.A.F. SIMA (Winnipeg, Manitoba)}

The incidence of intracranial meningioma in Manitoba, Canada, was reviewed from 1980 through 1987 . One hundred and ninety-three tumours were diagnosed in this period, corresponding to crude incidence rates of 2,3/100,000 for all meningiomas and $0,17 / 100,000$ for malignant meningiomas. The male:female ratio was $1: 1$ in malignant meningiomas, and 1:2 in the benign group. Meningotheliomatous 
tumours were the most common histopathological subtype (38\%), followed by transitional (33\%), unknown (8\%), malignant (7\%), fibroblastic $(7 \%)$, psammomatous $(4 \%)$, and angioblastic $(2 \%)$. Tumour definitions were based on W.H.O. criteria. Individuals with benign tumours were more likely to be without deficit $(26 \%)$ than their malignant counterparts (14\%). However, clinical features failed to conclusively differentiate the two groups. All malignant tumours demonstrated peritumoural edema, and they were the only type to display "mushrooming" and "fringing" on computed tomography.

33.

Cognitive Functioning in Treated Longterm Survivors with Highand Low-Grade Primary Brain Tumours - Preliminary Data

Y.M. ARCHIBALD, D. LUNN, D.R.MACDONALD, L. GASPAR, W. PEXMAN, R. DEL MAESTRO and J.G. CAIRNCROSS (London, Ontario)

Longer periods of survival and amelioration of symptoms have resulted from current treatment of patients with primary brain tumours. Nevertheless, diffuse cognitive deterioration has occasionally been described in some long-term survivors who have no known evidence of tumour progression, radiation necrosis or other identifiable processes. This study represents an attempt to document, in a systematic manner, the incidence, nature, extent and etiology of such decline.

Patients with high-grade tumours, who survive one year following surgical resection and subsequent therapy, are assessed at 6-month intervals on a battery of cognitive tests. Patients with low-grade tumours are assessed at yearly intervals from the time of initial assessment. All patients have a neurological exam and a CT scan within approximately 2 weeks of each cognitive assessment. Patients are followed for 3 years and then entered into a long-term follow-up protocol. The patient groups are compared with normal controls who receive the same assessment at similar time intervals.

The data show that on initial assessment, there is a significant difference in performance between high-grade tumour patients and their controls on most measures of intelligence, concentration, memory, and mental speed and fluency. However, there are fewer initial differences between the low-grade tumour patients and their controls.

Data from a retrospective patient group (followed before the start of the prospective study) provides information on cognitive functions over a more extended time period. For many patients, test performance remains stable from one assessment to the next, or shows only mild fluctuations. Nevertheless, some patients have shown a progressive deterioration in memory, with relative preservation of other cognitive functions. In addition, there are 3 patients, all with treated high-grade tumours, who have shown a dementia-like pattern of cognitive decline. The data thus suggest that cognitive deterioration may be expected to occur in some high-grade patients. Examples of representative neuropsychological test profiles will be presented.

34.

Incomplete Vascular Perfusion in Experimental Glial Tumours

C.L. FARRELL, C.R. FARRELL, R.F. DEL MAESTRO, P.A. STEWART and C. ELLIS (London, Ontario)

The flux of solutes into cerebral tissue is modulated by a number of factors including the flow of blood to the tissue and the permeabilitysurface area product of the microvessels within the tissue.

The purpose of this investigation was to characterize microvessel blood flow in the $\mathrm{C} 6$ astrocytoma spheroid implantation model and to determine the relationship of flow to the structure of the microvascular compartment.
Aquablak ink (AQ) was perfused to visualize all vessels not physically occluded to blood flow and horseradish peroxidase (HRP) was injected for 15 and 30 seconds to map out which vessels were perfused with blood. In normal cortical microvessels, the total vessel length measured in AQ perfused rats was $1093 \pm 49 \mathrm{~mm} / \mathrm{mm}^{3}$ compared to $222 \pm 15 \mathrm{~mm} / \mathrm{mm}^{3}$ in the tumour vasculature. Total vessel length in HRP perfused preparations was similar for cortex, but only half of the AQ value in tumours. Tumour vascular volume and surface area was approximately half the values in HRP specimens compared to AQ samples.

The data suggests that a proportion of tumour microvessels (up to $50 \%$ ) may not be actively perfused with blood. The reduced perfusion of brain tumour microvessels may result in a significant decrease in the area of interface available for blood-tissue exchange in tumours. Supported by: The Brain Research Fund Foundation

35.

Medullary Tumours; Presentation of Two Cases of the Pontomedullary Region

S.N. MARTINEZ, M. PARE, R. LEMAY, J. LESAGE and F. ROBERT (Montreal, Quebec)

Tumours of the medulla are more frequent in children than in adults. In general the treatment of medullary tumours is palliative. In a personal series of 142 posterior fossa tumours (S.N.M.) in adults, six originated from the medulla. There were four gliomas, one astrocytoma, and one hemangioblastoma. The latter two tumours presented exophitic growth characteristics.

The surgical treatment of exophitic tumours is warranted and has been attempted on our two patients. The first patient had a seven year survival and the second is alive at four years.

The case histories, investigation and evolution of these two patients is presented and the rational for the surgical treatment of these patients is discussed.

36.

Fractionated Postcraniotomy Light Delivery for Photodynamic Therapy of Malignant Brain Tumours

\section{P. MULLER and B. WILSON (Hamilton, Ontario)}

We have treated 56 patients with malignant brain tumours with intraoperative photodynamic therapy [PDT] using an argon dye pump laser and pre-operatively administered hematoporphyrin derivative or dihematoprophyrin ether. in 8 cases, in addition to cavitary photo-illumination, we have used cylindrical diffusion fibers to increase the amount of light energy administered to the tumour tissue intraoperatively. This interstitial photo-illumination was tolerated at light energy densities of less than $45 \mathrm{~J} / \mathrm{cm}$.

In our two most recent cases, both of whom had large bi-frontal malignant gliomas that crossed the midline in the corpus callosum and could not be illuminated adequately at a single session, cylindrical diffusion fibers were left in situ after intraoperative cavitary photo-illumination of the tumour residuum.

The fibers were protected from fracturing by placing all but the exposed diffusing end in a red rubber catheter of the appropriate diameter. The fibers were externalized through a separate stab wound as would be the case for a ventricular drain. Photo-illumination was continued one or two days post-operatively. The optimal fiber couple to the argon dye pump laser was achieved by assessing the fiber side scatter with a photometer.

The two patients received 3825 and 2475 Joules by intraoperative cavitary photo-illumination, respectively, for an energy density of 83 and $54 \mathrm{~J} / \mathrm{cm}^{2}$. The post-operative photo-illumination session resulted in the interstitial administration of 293 and $450 \mathrm{~J} / \mathrm{cm}$, respectively. The 
patient tolerated the fractionated photo-illumination well. A transient scalp inflammation occurred as the consequence of light transmission to skin from the implanted fibers.

37.

\section{Factors Affecting Tumour (T) Cisplatin (C) Levels (P)}

D. STEWART, A.M. MOLEPO, R. GREEN, H. HUGENHOLTZ, A. LAMOTHE, D. REDMOND, V. MONTPETIT and N. MIKHAEL (Ottawa, Ontario)

C $10 \mathrm{mg} / \mathrm{m}^{2}$ was given IV preoperatively to 45 consenting patients with T. $P$ were assayed by atomic absorption. Plasma-tissue transfer constants $(K)$ were calculated. $P$ was less $(p<.05)$ in gliomas $(G)$ and in extracerebral $T(E)$ than in primary brain lymphomas and meningiomas (L). $K$ was also less $(p<.05)$ in $G$ and $E$ than in $L$ and brain metastases (M). With respect to $T$ type ( $G$ vs adeno vs squamous $[S]$ vs others $[O]$, $P$ and $K$ for $G$ were $<0$ and $P$ for $S$ was $<0(p<.05)$. K but not $P$ decreased with time over $0.5-6 \mathrm{hr}$, suggesting (if confirmed) saturation of uptake (unlikely) or active extrusion of P. Pharmacokinetics did not affect $P$. For $G$ and M, P correlated with CAT scan edema intensity ( $p<$ $.05)$. Neither $K$ nor $P$ correlated with tumour size or location. $K$ increased with high serum potassium or calcium and in necrotic vs viable tumour $(p<.05)$. Hence, $T$ type may affect $P$ and $K$ more than $T$ location (E vs brain).

Supported by: OGH Foundation \& Health \& Welfare Canada
38.

Turcot's Syndrome: Case Report and Discussion of the Literature

H.B. NEWTON and M.G. MALKIN (New York, U.S.A.)

Turcot's Syndrome is a rare, genetically transmitted disease in which patients develop central nervous system malignancies and colonic polyposis. The inheritance pattern of Turcot's Syndrome remains unclear. Families with dominant and recessive inheritance, and sporadic cases have been described. We report a 26 year old man with apparent sporadic Turcot's Syndrome. He was well until 1986 when he developed intermittent abdominal discomfon. Sigmoidoscopy revealed numerous polyps, several of which showed carcinomatous change. Dukes' $\mathrm{C}$ colorectal carcinoma was diagnosed. Treatment consisted of total colectomy with construction of a Koch's pouch. He remained well for 3 years until onset of severe headache, nausea and projectile vomiting. CT scan disclosed a large, circumscribed, enhancing, right fronto-parietal mass with surrounding edema and ventricular compression. Neurological exam was unremarkable except for mild bilateral papilledema and left central facial weakness. After craniotomy and partial resection, pathological review disclosed anaplastic astrocytoma. Further treatment involved radiation therapy, $60 \mathrm{~Gy}$, by parallel opposed ports to the tumour bed. Chemotherapy has been initiated with BCNU, $200 \mathrm{mg} / \mathrm{m}^{2}$, IV every 8 weeks. Family history if negative for polyposis. One maternal grandfather died of pancreatic carcinoma, and one paternal grandmother died of an unspecified brain tumour. Cytogenetic, flow cytometric and oncogene analysis of the resected tumours, lymphocytes and fibroblast lines are proposed to characterize this patient's disease further. These results will be discussed in relation to the other 19 cases of Turcot's Syndrome reported in the literature. 